\title{
Editorial
}

\section{Cambio y continuidad 2.0}

Rayén Condeza Dall'Orso, Pontificia Universidad Católica de Chile, Santiago, Chile (rcondeza@uc.cl)

Con el título Cambio y continuidad: bienvenidos a Cuadernos.info (Valenzuela, 2013), Sebastián Valenzuela, entonces editor en jefe de Cuadernos.info, encabezóla publicación del número 32 de nuestra revista académica, actualmente adscrita a la Dirección de Investigación, Creación y Doctorado de la Facultad de Comunicaciones de la Pontificia Universidad Católica de Chile. En ese texto, que sugiero revisitar por la claridad relativa a la proyección de la revista allí explicada y su capacidad de enfocar lo esencial, a lo que Sebastián Valenzuela nos tiene acostumbrados, nuestro colega agradecía la labor de la editora saliente, María Olga del Piano, y daba la bienvenida a la nueva editora, Daniela Grassau, para liderar este proyecto de divulgación académica. Un proyecto que les fuera encargado por nuestra decana Silvia Pellegrini (2006-2016) y refrendado por nuestros decanos Eduardo Arriagada (2016-2020) y Paulina Gómez (2020-2024), en este pasar la posta que a mi juicio constituye una de las variables que inciden en la sustentabilidad y en la calidad de una publicación académica.

Hoy la revista forma "parte de las colecciones de SciELO, SCOPUS y Web of Science (Emerging Sources), entre otros, y Cuadernos.info se posicionó en el primer lugar del ranking Scimago de la región y el sexto a nivel iberoamericano. El factor de impacto de la revista se multiplicó al igual que su internacionalización" (Valenzuela \& Grassau, 2020, p. I). Sin duda, además del sólido trabajo de Sebastián Valenzuela, el aporte, la agudeza, generosidad y el compromiso dedicado a Cuadernos. info de Daniela Grassau han sido sustanciales para la mejora continua del proceso editorial y la calidad de los contenidos de esta publicación académica abierta, y de su reconocimiento creciente a nivel internacional en el campo multidisciplinar de las comunicaciones, con foco en Iberoamérica. 
A este equipo de cabeza y motor editorial, también compuesto por Constanza Vásquez, asistente editorial, así como por muchas otras personas reconocidas una a una en el editorial del número 47 Cambio, Despedida y Bienvenida (Valenzuela \& Grassau, 2020) les agradezco su acompañamiento y guía brindados durante el proceso de transición como editora entrante de Cuadernos.info, desde septiembre pasado. Han sido meses de aprendizaje invaluables y de avance en la comprensión vital de que el progreso de la ciencia es una tarea colectiva compleja.

Asimismo, agradezco la confianza depositada por nuestra actual decana Paulina Gómez (2020-2024) y por la Dirección de Investigación, Creación y Doctorado al solicitarme integrar parte del equipo de la revista y de contribuir en conjunto, desde esta nueva responsabilidad como editora en jefe, a proponer e implementar una nueva estructura para Cuadernos.info, que integre la participación de editores asociados, entre otras figuras. Se trata de continuar el camino que otros equipos editoriales abrieron para su desarrollo, incorporando los cambios pertinentes y, así, consolidar su impacto con sentido. En efecto, Cuadernos.info no solo busca contribuir en el ámbito de la comunidad científica disciplinar, un aporte reflejado en los índices de medición de calidad; al publicar investigaciones con impacto social y divulgar resultados de estudios relevantes, rigurosos, actuales, basados en la evidencia, también puede impactar a la sociedad en su conjunto. Como afirma Ramírez Montoya (2015), "hablar de una sociedad del conocimiento es hablar de vincular el saber con la aplicación del conocimiento" (p. 195), una necesidad apremiante para nuestra región y uno de los propósitos de los distintos equipos editoriales que, a lo largo del tiempo, han encabezado la revista. Por ello, el título Cambio y continuidad 2.0 decidido para el editorial de este número 48 , alude de manera directa a el del número 32. Espero que refleje las buenas prácticas que han guiado la labor de los equipos de trabajo a cargo de Cuadernos.info desde sus inicios, conjugándola con los nuevos rumbos que se estimen relevantes y necesarios de emprender.

En el año 2020, Cuadernos.info recibió 215 postulaciones y participaron 326 investigadores de muy variadas latitudes como evaluadores ciegos, con particular presencia del ámbito iberoamericano. Este proceso del flujo evaluativo es esencial para la calidad de toda revista. Parafraseando a Plata-Caviedes, Córdoba-Salgado y Trzesniak (2012), los revisores proporcionan a los editores los recursos para tomar una decisión fundamentada sobre la publicación de un artículo. Fue esta fase, precisamente, una de las que se vio más afectada por la pandemia de COVID19. Agradecemos a los autores y evaluadores por su disponibilidad y capacidad de resiliencia, en un contexto de cambio e incertidumbre únicos, además de su comprensión cuando los tiempos de respuesta y de decisión editorial se vieron tensionados. 
La propagación mundial de esta nueva enfermedad ha afectado a millones de personas en muy diversos planos, entre los cuales se incluye una parte importante de los integrantes de la comunidad y del espacio de diálogo en el ámbito de las comunicaciones al que Cuadernos.info busca contribuir. En nombre del equipo editorial, me permito expresar nuestras más sinceras condolencias hacia los colegas que perdieron a algún ser querido, así como nuestra solidaridad con quienes atraviesan momentos difíciles por esta causa.

Este mismo contex to ha demostrado la importancia que reviste la investigación en comunicaciones en la actualidad, así como la necesidad de reflexionar en torno a la casa común que compartimos y a su cuidado, con una conciencia renovada. Es más que nunca necesario contar con conocimiento emanado de procesos rigurosos de reflexión, interrogación y de estudio en torno a las actuales problemáticas en el área de las comunicaciones, como a los que apunta Cuadernos.info. Martín Sempere (2001, citando a Van Raan, 1997) advierte al respecto que "la garantía de calidad debe ser y seguirá siendo la cuestión más esencial en la comunicación científica” (p. 7). Por lo mismo, es mi propósito persistir en este objetivo. También suscribo a la afirmación de Gutiérrez San Miguel, Tur-Viñes y Fonseca- Mora (2016) y a la importancia de adherir a los estándares de ética internacionales, como los recomendados por COPE: "La búsqueda de una ética editorial se fusiona con el proceso de calidad de las revistas como medio para mejorar y validar los textos científicos que se publican" (p. 54).

Un primer cambio a partir de este número 48 es la migración a la nueva plataforma OJS 3.02, desde el mes de enero de 2021. Los invito a darse de alta en este nuevo espacio de interacción, que esperamos permita mejorar los flujos del proceso editorial, los procesos de evaluación y de selección, así como optimizar los tiempos de respuesta a los autores. Esta mejora es apoyada por el servicio de soporte a la gestión editorial del Sistema de Bibliotecas (SIBUC) de la Pontificia Universidad Católica de Chile a su Red de revistas científicas, para su edición y publicación electrónicas (Didier, 2019). El proyecto, financiado por el Fondo de Publicación de Revistas Científicas del Programa de Información Científica de la Agencia Nacional de Investigación y Desarrollo (ANID) de Chile, incluye el servicio de detección de similitudes y su marcaje en SciELO. El equipo editorial de Cuadernos. info forma parte del comité editorial de revistas académicas de la universidad, en el camino al desarrollo de un repositorio institucional. Este soporte crucial de SIBUC ha permitido a Cuadernos.info reforzar uno de los principios que la guían, y que según Alperín y Fischman (2015) comparten las revistas de la región, en torno a la noción de la información científica como un bien común y del consecuente acceso libre a esa información, sin fines de lucro. 
El año 2021 marcará también la concreción del aumento en la frecuencia de publicación de Cuadernos.info, de dos a tres números por año (enero-mayoseptiembre), dos dedicados a temas generales y uno, monográfico. La exitosa convocatoria a proposiciones de dossiers nos permitió realizar un desafiante proceso de preselección y de dictamen final de los equipos de editores invitados, cuyas convocatorias serán publicadas próximamente, para 2022 y 2023.

Las diez contribuciones de este nuevo número de Cuadernos.info, dedicado a Temas Generales, fueron editadas por Daniela Grassau. Muestran una paleta variada de estudios desarrollados por autores de Colombia, Chile, España y Portugal. Coinciden en estudiar casos en los que el ecosistema de medios digital coexiste con los medios tradicionales y el offline. Representan el trabajo de 19 investigadoras e investigadores, afiliados a quince universidades iberoamericanas.

El primer artículo, “Tendencias de los artículos académicos sobre TIC y web social en el período 2013-2017", un estudio cuantitativo de Valeriano Piñeiro-Navaly Ricardo Morais, cartografía las publicaciones académicas en comunicaciones de España e Hispanoamérica en torno a las tecnologías de información y de las comunicaciones y de la web 2.0 indexadas en Scimago Journal \& Country Rank, a partir de siete variables de agrupación (origen geográfico, idioma, medios protagonistas, disciplinas, financiación, tipologías de trabajo y paradigmas epistemológicos).

La segunda y tercera contribuciones investigan experiencias de comunicación política. Silvia Marcos-García, Laura Alonso-Muñoz y Amparo López-Meri analizan en su artículo las "Campañas electorales y Twitter. La difusión de contenidos mediáticos en el entorno digital” la incorporación de esta red social por los cuatro partidos políticos más votados en España durante la campaña electoral de 2016, en un contexto híbrido de comunicación política. A partir de un análisis cuantitativo de los mensajes con mayor impacto de los perfiles de dichos conglomerados en Twitter, así como los difundidos durante los días de máxima actividad de cada partido, observan la asociación entre el uso de Twitter y la presencia de los líderes en los medios tradicionales, escenario donde el debate televisado cobra una importancia particular.

En el tercer manuscrito, "Uso intensivo de herramientas y recursos de profesionalización política en campañas presidenciales: el caso de Colombia 2018", de los autores Néstor Julián Restrepo Echavarría y Luis Antonio González Tule, se discuten los resultados de la aplicación de un instrumento que midió los índices de profesionalización de las campañas electorales de los tres candidatos principales a la presidencia de Colombia, incluyendo dimensiones como la comunicativa, los medios empleados, el trabajo de proyección de la imagen de los líderes en carrera y el empleo del recurso de la emotividad de la puesta en escena. 
En el ámbito de los estudios culturales, Andrés Maximiliano Tello, Consuelo Dinamarca Noack y Sebastián Escobar Pulgar se aproximan, en el cuarto manuscrito, a las actuales dinámicas de cambio cultural en Chile, en particular a partir del uso de las licencias Creative Commons en el contexto de la cultura libre en Internet y de la relación percibida con las nuevas formas de producir, hacer circular y consumir obras en el campo cultural de ese país. Los autores profundizan en el fenómeno a partir de entrevistas cualitativas a agentes culturales de editoriales independientes y netlabels nacionales.

Los BookTubers, erigidos como mediadores de lectura entre los jóvenes y los adolescentes, como afirma su autor, constituyen el foco del quinto artículo. Aplicando una metodología mixta, en el artículo que lleva por título "Nuevos mediadores de la LIJ: análisis de los BookTubers más importantes de habla hispana”, Anastasio García-Roca describe quiénes son estos influenciadores, así como la variedad temática de sus propuestas de contenido audiovisual, en una plataforma particular, en la que predominan las BookTubers y determinados temas que interesan a estos referentes sociales para las nuevas generaciones.

Siempre en el ámbito de la nueva industria cultural, con predominio actual de plataformas como Netflix, Mayte Donstrup y Cristina Algaba conducen en el sexto artículo un análisis crítico del discurso ideológico movilizado en dos series emitidas a partir de 2019: The Society y The Passage. Su artículo "La dualidad en la ficción televisiva fantástica" se aproxima al estudio de las ideologías presentes en sus narrativas como un objeto de reflexión en torno a las actuales sociedades que habitamos.

La publicidad es otro de los ejes mediáticos de interés para la revista. Los artículos siete y ocho remiten precisamente a estudios del discurso publicitario, desde perspectivas de épocas y contextos de análisis distintos en España. "Publicidad, diversidad fenotípica y dinamización intercultural en Cataluña en tiempos de crisis y cambios", de Nicolás Lorite García, examina cómo el discurso publicitario posiciona una imagen de identidad en los tiempos de crisis económica en Cataluña y de tendencia hacia su independencia de España (2014). Para ello, analiza 443 spots de diferentes marcas emitidos por la cadena de mayor audiencia en dicho territorio, con hallazgos en torno a una imagen cultural blanco-caucásica predominante y excluyente de la diversidad cultural entonces imperante.

La octava publicación, de María Arroyo Cabello, "Génesis de la publicidad técnica en España. El caso de El Bazar Murciano (1892-1929)" sostiene que la aparición de la publicidad técnica en España es anterior a la fechada hasta ahora, en el primer tercio del siglo XX. Lo argumenta a partir de un análisis de contenido cualitativo de la publicación de la tienda de El Bazar Murciano (1892-1929). A partir 
de sus hallazgos, afirma que dicha publicación comercial fue pionera en aplicar el contenido de marca y que respondió a una estrategia publicitaria primaria en el ámbito de los llamados house organs editados en ese país.

Las dos siguientes investigaciones se sitúan en el escenario universitario. El artículo nueve "Competencias profesionales para la gestión de emisoras universitarias en España”, de Miguel Ángel Ortiz Sobrino, Carmen Marta-Lazo y Paloma Contreras-Pulido, explora la percepción de los directores de 30 radios universitarias sobre las competencias profesionales necesarias para su operación, mediante un estudio mixto. Específicamente, analizan las competencias declaradas para los puestos de dirección de la emisora, coordinadores de programación, técnico de producción y directores de programas.

Finalmente, el artículo de Nelson Nieto Borda describe los resultados de una investigación que se propuso sistematizar una experiencia reciente de formación universitaria en periodismo en Bogotá, durante los años 2016 y 2017. El manuscrito "Enseñanza del periodismo transmedia en Colombia, una experiencia pedagógica con estudiantes universitarios" describe con detalle la metodología de aprendizaje basado en proyectos, empleada para lograr un aprendizaje significativo e integral de los estudiantes. Recoge las percepciones de estos participantes, quienes fueron protagonistas en la toma de decisiones para crear un proyecto periodístico transmedia, acercándose así al ecosistema mediático actual, que forma parte del escenario multiplataforma de su futuro desempeño profesional.

Los invito a imbuirse en esta interesante selección de publicaciones que enriquecen a Cuadernos.info y, a través de su lectura y divulgación, el campo de la investigación en comunicaciones en Iberoamérica.

RAYÉN CONDEZA DALL'ORSO, PHD

Editora en jefe

Cuadernos.info 


\section{REFERENCIAS}

Alperín, J. P. \& Fischman, G. (Eds). (2015). Hecho en Latinoamérica: acceso abierto, revistas académicas e innovaciones regionales (Made in Latin America Open access, academic journals, and regional innovations). Buenos Aires: Clacso.

Didier, E. (IR) (2019). Mejoramiento de la gestión editorial de las Revistas UC y su visibilidad en el entorno digital mediante una plataforma de acceso, articulación, colaboración, servicios de difusión y marketing científico (FP 190007-2019) (Improvement of the editorial management of UC Journals and their visibility in the digital environment through a platform for access, articulation, collaboration, dissemination services and scientific marketing (FP190007-2019)). Santiago de Chile: Concurso Fondo de Publicación de Revistas científicas, convocatoria 2019, Agencia Nacional de Investigación y Desarrollo (ANID).

Gutiérrez San Miguel, B., Tur-Viñes, B., \& Fonseca- Mora. M. C. (2016). Principios éticos de los editores en las revistas científicas españolas de Comunicación, Educación y Psicología (Ethical principles of publishers in the Spanish scientific journals on Communication, Education and Psychology). Ibersid, 10(2), 53-60. Retrieved from https://www.ibersid.eu/ojs/index.php/ibersid/article/view/4310

Martín Sempere, M.J. (2001). Papel de las revistas científicas en la transferencia de conocimientos (Role of academic journals in knowledge transfer). En Román Román, A (Coord.) La edición de revistas científicas. Guía de buenos usos (pp. 7-10). Madrid: Centro de Información y Documentación Científica CINDOC (SCIC).

Plata-Caviedes, T., Córdoba-Salgado, O. A., \& Trzesniak, P. (2012). Dictámenes en Revistas Científicas: lo que necesitan los editores y autores, lo que hacen los evaluadores (Journal referee reports: What editors and authors need, what referees do). Revista Colombiana de Psicología, 21(1),37-55. Retrieved from https://revistas.unal.edu.co/index.php/psicologia/article/view/31031

Ramirez-Montoya, M. S. (2015). Acceso abierto y su repercusión en la sociedad del conocimiento: reflexiones de casos prácticos en Latinoamérica (Open access and its impact on the knowledge society: reflections of practical cases in Latin America). Education in the Knowledge Society, 16(1), 103-118. Retrieved from https://revistas.usal.es/index.php/eks/article/view/eks2015161103118

Valenzuela, S. (2013). Editorial: Cambio y continuidad: Bienvenidos a Cuadernos.info (Editorial: Change, Continuity and Welcome to Cuadernos.info). Cuadernos.info, (32), I-III. Retrieved from https://cuadernos.info/index.php/CDI/article/view/501/464

Valenzuela, S. \& Grassau, D. (2020). Editorial: Cambio, despedida y bienvenida (Editorial: Change, farewell and welcome). Cuadernos.info, 47, I-III. https://doi.org/10.7764/cdi.47.2176 